https://doi.org/10.22319/rmcp.v12i2.5466

Nota de investigación

\title{
Predicción de la calidad fermentativa de ensilados de girasol mediante espectroscopía de reflectancia en el infrarrojo cercano (NIRS) sobre muestras secas
}

Sonia Pereira-Crespo ${ }^{\text {a }}$

Aurora Sainz-Ramírez ${ }^{\mathrm{b}}$

Dalia Andrea Plata-Reyes ${ }^{b}$

Aida Gómez-Miranda ${ }^{b}$

Felipe González-Alcántara ${ }^{\text {b }}$

Adrián Botana ${ }^{a}$

Laura González ${ }^{\text {a }}$

Marcos Veiga $^{\text {a }}$

Cesar Resch $^{\mathrm{a}}$

Roberto Lorenzana ${ }^{c}$

Fernando Próspero-Bernal a*

Carlos Manuel Arriaga-Jordán ${ }^{\mathrm{b}}$

Gonzalo Flores-Calvete ${ }^{a}$

${ }^{a}$ Centro de Investigacións Agrarias de Mabegondo de la Axencia Galega da Calidade Alimentaria de la Consellería do Medio Rural. Xunta de Galicia, Mabegondo, Abegondo, A Coruña, Galicia, España.

${ }^{\mathrm{b}}$ Universidad Autónoma del Estado de México, Instituto de Ciencias Agropecuarias y Rurales. Toluca, Estado de México, México.

${ }^{\mathrm{c}}$ Laboratorio Interprofesional Galego de Análise do Leite, Mabegondo, Abegondo, A Coruña, Galicia, España. 
*Autor de correspondencia: fer_104_7@ @otmail.com

\section{Resumen:}

El objetivo del presente trabajo fue evaluar la capacidad de predicción de las ecuaciones de calibración desarrolladas mediante NIRS (espectroscopía de reflectancia en el infrarrojo cercano) sobre muestras secas y molidas, para estimar la calidad fermentativa de ensilados de girasol. Un total de 52 muestras de ensilados procedentes de diferentes ensayos de silos de laboratorio realizados en el CIAM (Centro de Investigacións Agrarias de Mabegondo), cuyo espectro NIRS se registró sobre muestras secas en estufa y molidas. Las muestras en estado fresco fueron analizadas por métodos de referencia. Se determinó el pH, ácido láctico, ácido acético, etanol, nitrógeno amoniacal y nitrógeno soluble. Las calibraciones NIRS fueron desarrolladas utilizando regresión por mínimos cuadrados parciales modificada, realizando la regresión entre los datos espectrales y los de referencia. La capacidad predictiva de las ecuaciones obtenidas osciló entre excelente y buena, mostrando coeficientes de determinación de validación cruzada $\left(\mathrm{r}^{2}{ }_{\mathrm{vc}}\right)$ iguales o superiores a 0.88 . Los valores del índice RPD para todos los parámetros estudiados fueron iguales o superiores a 3.0, por lo tanto, las ecuaciones de calibración obtenidas sobre muestras secas y molidas pueden utilizarse satisfactoriamente para predecir la calidad fermentativa de ensilados de girasol en análisis de rutina.

Palabras clave: Forrajes, Parámetros de fermentación, Espectroscopía de reflectancia.

Recibido: 23/08/2019

Aceptado:18/04/2020

La evaluación nutritiva de los forrajes es relevante debido a la elevada variabilidad de su valor nutritivo y a la alta contribución al total de la materia seca de las raciones de ganado, en comparación con el concentrado. El valor nutricional del ensilado está condicionado fundamentalmente, además de por las características intrínsecas del forraje en el momento del corte, por la calidad de fermentación desarrollada durante el almacenamiento en el silo ${ }^{(1)}$, siendo sumamente variable dependiendo de la ensilabilidad del forraje y del tratamiento post$\operatorname{cosecha}^{(2)}$, y afectando particularmente al valor nitrogenado y la ingestión voluntaria del ensilado $^{(3)}$. Por tanto, para un uso eficiente de los ensilados es necesario caracterizar previamente su calidad fermentativa, para lo cual es fundamental disponer de métodos rápidos, precisos y fiables. Los análisis instrumentales para determinar los parámetros de calidad fermentativa de un ensilado son complejos, costosos y requieren un gran consumo de tiempo. 
La tecnología NIRS (Espectroscopía de reflectancia en el infrarrojo cercano) está ampliamente reconocida como una técnica analítica rápida, barata y de gran precisión en la caracterización de la calidad de conservación de los ensilados, como alternativa al análisis por vía húmeda ${ }^{(4)}$, y además, se trata de una tecnología medioambientalmente limpia que no emplea reactivos ni genera residuo alguno. El análisis NIRS de los ensilados en modo intacto, en fresco, implica gran dificultad, debido a que se trata de un material con elevada heterogeneidad ${ }^{(5)}$. Por otro lado, la presencia de agua en la muestra intacta interfiere en el espectro NIRS, dado que ésta absorbe parte de la radiación infrarroja, generando dos bandas de absorción muy significativas en el espectro. No obstante, hay que señalar que el análisis NIRS de muestras secas presenta desventajas respecto al análisis con muestras frescas, debido a que los constituyentes volátiles del ensilado, como los ácidos de fermentación, alcoholes y amonio, son liberados y perdidos durante el proceso de secado de la muestra. En un estudio se determinó por métodos de referencia una serie de muestras antes y después del proceso de secado, y se comparó la predicción de las ecuaciones NIRS desarrolladas sobre muestras secas con las realizadas con material húmedo, como resultado se observó que la predicción del $\mathrm{pH}$, láctico y nitrógeno amoniacal fue más robusta sobre material seco mientras que la calidad de la predicción para el acético fue mejor cuando la medida NIRS se realizaba sobre la muestra húmeda ${ }^{(6)}$. Esto se atribuye a que la calidad de predicción obtenida para los diferentes parámetros por los dos métodos no tiene relación con las pérdidas durante el secado de las muestras, toda vez que la reducción de la concentración de ácido láctico, ácido acético, etanol y nitrógeno amoniacal en la materia seca durante el secado fue del $3.5 \% ; 57 \%, 53 \%$ y $100 \%$ para ensilados de hierba y del $3.5 \% ; 83 \%, 16 \%$ y $100 \%$ para ensilados de maíz, respectivamente, en clara correspondencia con su volatilidad ${ }^{(6)}$.

Por otra parte, en otro trabajo evaluaron el efecto de la preparación de la muestra de ensilados de maíz (estado fresco $v s$ seca y molida) sobre la estimación de parámetros fermentativos mediante NIRS, y los resultados indican que las muestras en estado fresco proporcionan una capacidad predictiva ligeramente superior para ácido acético $\left(\mathrm{r}^{2}{ }_{\mathrm{vc}}=0.85\right.$ vs 0.82$)$ y ácido láctico $\left(\mathrm{r}_{\mathrm{cv}}^{2}=0.78 v s 0.73\right)$ e inferior para $\mathrm{pH}\left(\mathrm{r}_{\mathrm{vc}}^{2}=0.54 v s 0.63\right)^{(7)}$. Un estudio realizado en el Centro de Investigacións Agrarias de Mabegondo (CIAM) en Galicia, indica la conveniencia de utilizar las muestras secas y molidas en vez de intactas, al obtener modelos de predicción de la composición química y calidad fermentativa de ensilados de hierba con mayor precisión ${ }^{(8)}$. En otro trabajo realizado recientemente en el CIAM se evaluó la predicción de parámetros fermentativos de ensilados de hierba mediante calibraciones NIRS, desarrolladas sobre material seco y molido, los resultados obtenidos fueron satisfactorios con coeficientes de determinación iguales o superiores a $0.80^{(9)}$. El conocimiento de la calidad fermentativa de nuevos tipos de forrajes de manera rápida y precisa requiere avanzar en el desarrollo de nuevas calibraciones NIRS, en ese sentido, el objetivo del presente trabajo fue evaluar la capacidad predictiva de las ecuaciones de calibración NIRS en muestras secas y molidas para estimar parámetros de calidad fermentativa de ensilados de girasol. 
El trabajo se realizó con un total de 52 muestras de ensilados de girasol provenientes de diferentes ensayos de silos de laboratorio realizados en el CIAM durante los años 2016 y 2017. La colección de muestras abarca una elevada variabilidad en cuanto al estado de madurez, incluye muestras de girasol cosechadas en diferentes estados fenológicos, según la escala de Schneiter y Miller, desde el estado R4 (1 semana antes de la floración) hasta el estado R7 (5 semanas tras el inicio de la floración) ${ }^{(10)}$. El forraje con el que se elaboraron los silos de laboratorio procede del cultivo de dos híbridos comerciales: una variedad forrajera (Rumbosol 91) y una variedad de aceite (ES Shakira), realizado en las fincas experimentales del CIAM situadas en dos localidades de Galicia (España), en Mabegondo (zona costera atlántica noroccidental de Galicia, a 100 msnm) y en Pobra de Brollón (zona interior de Galicia, a $400 \mathrm{msnm}$ ). Además, en los ensayos realizados se incluyeron ensilados sin aditivo y con diferentes aditivos: ácido fórmico y dos inoculantes comerciales (uno de ellos a base de bacterias lácticas homofermentativas y otro a base de bacterias lácticas homo y heterofermentativas).

Los silos de laboratorio se abrieron a los 60 días tras su realización. Las muestras de ensilados, tras homogeneizarlas manualmente, se dividieron en dos alícuotas, una de las cuales se secó en estufa a $80{ }^{\circ} \mathrm{C}$ durante $16 \mathrm{~h}^{(11)}$ y la otra se congeló a $-18{ }^{\circ} \mathrm{C}$, envasada herméticamente al vacío en envases de plástico, hasta la realización de su análisis fermentativo por métodos de referencia. La información espectral de las muestras secas y molidas a $1 \mathrm{~mm}$, se obtuvo en un espectrofotómetro monocromador Foss NIRSystem 6500 (Foss NIRSystem, Silver Spring, Washington, USA), situado en una sala con temperatura controlada $\left(24 \pm 1^{\circ} \mathrm{C}\right)$ y provisto de módulo de giro que realiza medidas de reflectancia (R) en la región espectral comprendida entre 400 y 2,500 nm, a intervalos de $2 \mathrm{~nm}$. Los datos de absorbancia son expresados como $\log (1 / \mathrm{R}, \mathrm{R}=\mathrm{Reflectancia).} \mathrm{La} \mathrm{recogida} \mathrm{de} \mathrm{espectros} \mathrm{y} \mathrm{el}$ análisis quimiométrico de los datos se llevó a cabo mediante el programa Win ISI II v.1.5 (Infrasoft International, Port Matilda, PA, USA) ${ }^{(12)}$. Mediante el algoritmo CENTER $^{(13)}$, se realizó un Análisis de Componentes Principales (ACP), seguido del cálculo de distancias entre espectros en un espacio n-dimensional a través de la distancia de Mahalanobis, el cual permitió estudiar la estructura y variabilidad espectral de la población y detectar muestras anómalas ${ }^{(13)}$. La distancia Global de Mahalanobis (GH) está definida como la distancia entre una muestra y el centro de la población en el espacio definido por el ACP (Figura 1), considerando como muestras atípicas aquellas con valores GH>3 (anómala espectral) ${ }^{(13)}$. 
Figura 1: Representación tridimensional de los espectros del colectivo de muestras según la distancia Global de Mahalanobis

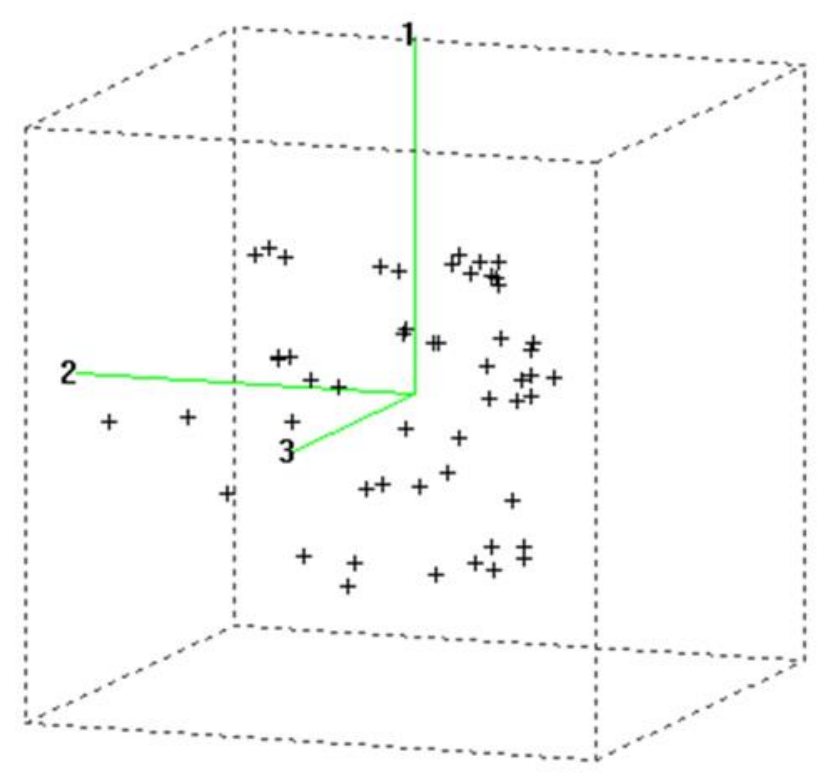

Se aplicó a los datos espectrales el pretratamiento SNV-Detrend ${ }^{(14)}$ para corregir el fenómeno de radiación dispersa y se evaluaron los ocho tratamientos matemáticos siguientes: $1,5,5,1$; $1,6,4,1 ; 1,10,5,1 ; 1,10,10,1 ; 2,5,5,1 ; 2,6,4,1 ; 2,10,5,1 ; 2,10,10,1$, donde el primer dígito expresa el orden de la derivada ( $1=$ primera derivada, $2=$ segunda derivada), el segundo dígito indica el tamaño del segmento sobre el cual se realiza la derivación (intervalo expresado en nanómetros), el tercer y cuarto dígito indican el tamaño de los intervalos, expresados en nanómetros, empleados para el cálculo de suavización de la señal ${ }^{(15)}$.

El desarrollo de las ecuaciones de calibración se realizó mediante regresión por mínimos cuadrados parciales modificada (MPLS) ${ }^{(16)}$ entre los datos espectrales y los de referencia, incluyendo cuatro grupos de validación cruzada para prevenir el sobreajuste, que fueron secuencialmente utilizados para efectuar la validación de las ecuaciones generadas.

El análisis fermentativo de las muestras intactas de ensilados se realizó mediante métodos de referencia, por duplicado ${ }^{(17)}$. Sobre un extracto de $50 \mathrm{~g}$ de muestra fresca de ensilado, macerada a temperatura ambiente durante $2 \mathrm{~h}$ en $150 \mathrm{ml}$ de agua destilada, se determinó el $\mathrm{pH}$, el nitrógeno amoniacal $\left(\mathrm{N}-\mathrm{NH}_{3}\right)$ con un electrodo selectivo (Orion) y el nitrógeno soluble (N-sol) mediante digestión macro Kjeldahl. Los ácidos de fermentación (láctico, acético y propiónico) y el etanol se determinaron por cromatografía de gases (Agilent Technologies, USA) con una columna capilar de elevada polaridad BR-SwaxAcids (30 m x 0.53 mm x 1 
$\mu \mathrm{m}$; Bruker, USA). Los parámetros de $\mathrm{N}^{-\mathrm{NH}_{3}}$ y N-sol se refirieron al nitrógeno total y los ácidos de fermentación y el etanol a materia seca.

Los estadísticos utilizados para seleccionar las mejores ecuaciones de calibración fueron los errores estándar de calibración (EEC) y de validación cruzada (EEVC) y los coeficientes de determinación $\left(\mathrm{r}_{\mathrm{c}}^{2} \mathrm{y} \mathrm{r}_{\mathrm{vc}}^{2}\right)$ obtenidos en el proceso de calibración y validación cruzada, respectivamente ${ }^{(18)}$. Además, se utilizaron otros estadísticos de gran utilidad para evaluar la capacidad predictiva de las ecuaciones de calibración obtenidas, como el índice RER o relación entre el rango de los datos de referencia y el EEVC y el índice RPD o relación entre la desviación estándar de los datos de referencia y el EEVC ${ }^{(19)}$.

Las características descriptivas (rango, media y la desviación estándar) de los parámetros fermentativos del colectivo de calibración se muestran en el Cuadro 1, observándose un amplio rango y una elevada desviación estándar, para cada uno de los componentes analizados por métodos de referencia. Esta elevada variabilidad confirma que dicho colectivo está constituido por ensilados muy diversos, factor clave para la obtención de ecuaciones de calibración robustas ${ }^{(20)}$. El valor medio (y rango de variación) del contenido en materia seca de la población de ensilados que conformaron el set de calibración fue de $16.0 \%$ (11.3 a $21.9 \%)$.

Cuadro 1: Rango, media y desviación estándar de los parámetros de calidad fermentativa del grupo de calibración $(n=52)$ de ensilados de girasol

\begin{tabular}{lcccc}
\hline Parámetro & \multicolumn{2}{c}{ Rango } & Media & DE \\
\hline $\mathrm{pH}$ & 3.55 & 4.29 & 3.91 & 0.21 \\
Láctico, \%MS & 0.00 & 15.74 & 7.99 & 5.51 \\
Acético, \%MS & 0.52 & 4.04 & 2.39 & 1.10 \\
Etanol, \%MS & 0.90 & 12.50 & 3.78 & 3.43 \\
$\mathrm{~N}^{-\mathrm{NH}_{3}, \% \mathrm{NT}}$ & 2.21 & 10.37 & 6.09 & 2.59 \\
$\mathrm{~N}$ soluble, \%NT & 26.96 & 52.95 & 41.41 & 7.68 \\
\hline
\end{tabular}

$\mathrm{MS}=$ materia seca; $\mathrm{N}-\mathrm{NH}_{3=}$ nitrógeno amoniacal; $\mathrm{NT}=$ nitrógeno total; $\mathrm{DE}=$ desviación estándar.

En el Cuadro 2 se muestran los estadísticos de las ecuaciones de calibración obtenidas para la predicción de los parámetros de calidad fermentativa. Los coeficientes de determinación en el proceso de validación cruzada $\left(\mathrm{r}^{2} \mathrm{vc}\right)$ ofrecen información sobre la calidad de la calibración, del cual se han definido tres niveles de precisión de los modelos de predicción, donde valores de $\mathrm{r}_{\mathrm{cv}}^{2}$ superiores a 0.90 indican una capacidad de predicción excelente, valores 
de $r^{2}$ cv entre 0.89 y 0.70 indican que la calibración es considerada de buena capacidad de predicción cuantitativa, y calibraciones con valores de $r^{2}{ }_{c v}$ entre 0.69 y 0.50 permiten sólo una adecuada discriminación entre valores altos, medios y bajos ${ }^{(21)}$. Por tanto, los valores de $\mathrm{r}^{2}{ }_{\mathrm{vc}}$ para los parámetros de $\mathrm{pH}\left(\mathrm{r}_{\mathrm{vc}}^{2}=0.98\right), \mathrm{N}-\mathrm{NH}_{3}\left(\mathrm{r}^{2}{ }_{\mathrm{vc}}=0.96\right)$, acético $\left(\mathrm{r}^{2}{ }_{\mathrm{vc}}=0.94\right)$, láctico $\left(\mathrm{r}^{2}{ }_{\mathrm{vc}}=0.90\right)$ y etanol $\left(\mathrm{r}^{2}{ }_{\mathrm{vc}}=0.90\right)$ indican una capacidad de predicción excelente, y el contenido en $\mathrm{N}$ soluble $\left(\mathrm{r}^{2}{ }_{\mathrm{vc}}=0.88\right)$ presenta una capacidad de precisión buena ${ }^{(21)}$. La exactitud de la predicción puede juzgarse en función de los valores de los índices RER y RPD ${ }^{(19)}$, dado que valores de RPD mayores de 3 y de RER mayores de 10 son tomados como indicadores de la utilidad de las predicciones ${ }^{(19)}$. La elevada desviación estándar y el amplio rango de variación del colectivo de calibración explican los adecuados valores del RPD $(3.0-6.5)$ y RER $(9.0-22.8)$ obtenidos.

Cuadro 2: Estadísticos de la ecuación de calibración desarrollada para la predicción de los parámetros de calidad fermentativa de ensilados de girasol

\begin{tabular}{|c|c|c|c|c|c|c|c|}
\hline Parámetro & $\mathbf{T M}$ & EEC & $\mathbf{r}^{2}$ & EEVC & $\mathbf{r}^{2} \mathrm{vc}$ & RER & RPD \\
\hline $\mathrm{pH}$ & $(1,5,5,1)$ & 0.02 & 0.99 & 0.03 & 0.98 & 22.8 & 6.5 \\
\hline Láctico, \%MS & $(2,10,10,1)$ & 1.63 & 0.91 & 1.75 & 0.90 & 9.0 & 3.2 \\
\hline Acético, \%MS & $(2,10,5,1)$ & 0.17 & 0.97 & 0.25 & 0.94 & 13.9 & 4.3 \\
\hline Etanol , \%MS & $(2,6,4,1)$ & 0.97 & 0.92 & 1.07 & 0.90 & 10.9 & 3.2 \\
\hline $\mathrm{N}-\mathrm{NH}_{3}, \% \mathrm{NT}$ & $(2,10,10,1)$ & 0.44 & 0.97 & 0.54 & 0.96 & 15.1 & 4.8 \\
\hline $\mathrm{N}$ soluble, $\% \mathrm{NT}$ & $(2,10,10,1)$ & 2.18 & 0.92 & 2.58 & 0.88 & 10.1 & 3.0 \\
\hline
\end{tabular}

Las ecuaciones de predicción para $\mathrm{pH}$, ácido acético, etanol, $\mathrm{N}-\mathrm{NH}_{3}$ y $\mathrm{N}$-sol presentan valores de RPD>3 y RER >10, cumpliendo con los valores recomendados en la bibliografía ${ }^{(19)}$. Así, el valor de $\mathrm{pH}$ sería el estimado con mayor exactitud (RER=22.8; $\mathrm{RDP}=6.5$ ) seguido por los valores de ácido acético (RER=19.5; RDP=4.3), $\mathrm{N}-\mathrm{NH}_{3}$ (RER=19.5; RDP=4.3), etanol (RER=10.9; RDP=3.2) y N-sol (RER=10.1; RDP=3.0). En el caso de la ecuación de predicción del ácido láctico el valor del índice RER (9.0) no alcanzó el valor recomendado, si bien el valor de RPD (3.2) supera el valor mínimo recomendado en la bibliografía ${ }^{(19)}$. Por lo tanto, los valores de los estadísticos RER y RPD confirman la elevada exactitud y precisión de las ecuaciones obtenidas, asegurando la validez de estas desde el punto de vista de su aplicación en análisis cuantitativo ${ }^{(19)}$. 
La información existente en la bibliografía acerca de la aplicabilidad de la técnica NIRS para la predicción del $\mathrm{pH}$ de ensilados de girasol forrajero es escasa ${ }^{(22)}$. Un trabajo realizado con un colectivo similar al de este trabajo, una colección de 50 muestras secas y molidas de ensilados experimentales de girasol, cuyo resultado mostró una menor capacidad predictiva para la estimación del $\mathrm{pH}$ que en el presente trabajo, con valores inferiores de $\mathrm{r}^{2}{ }_{\mathrm{vc}}(0.86)$, RER (5.9), RPD (2.5) y mayor de EEVC $(0.44)^{(22)}$. En otros trabajos, sobre muestras en estado fresco, han obtenido una menor capacidad predictiva para el valor de $\mathrm{pH}$ que en este trabajo, con valores de $\mathrm{r}^{2}$ vc de $0.85,0.72$ y 0.78 en ensilados de hierba ${ }^{(4)}$, ensilados de cebada $^{(23)}$ y ensilados de raigrás ${ }^{(24)}$, respectivamente.

Respecto al contenido en ácido láctico, ácido acético y etanol, sobre muestras frescas de ensilado de hierba, han indicado una precisión menor a la observada en este estudio, con valores de $r^{2}$ vc y RPD de 0.83 y $2.5,0.73$ y 2.0 y 0.77 y 2.8 , respectivamente ${ }^{(25)}$. Se ha reportado en ensilado de hierba, sobre muestras frescas, valores de $\mathrm{r}^{2}{ }_{\mathrm{vc}}$ y RPD para $\mathrm{N}$-sol de 0.89 y 3.3 , y de 0.92 y 4.0 para $\mathrm{N}-\mathrm{NH}_{3}{ }^{(25)}$, valores similares a los obtenidos en este estudio.

Una vez elaborado los modelos de predicción se debe evaluar el ajuste de los datos al mismo, para lo cual se utiliza la representación gráfica de los valores predichos mediante NIRS versus los valores de referencia. En la Figura 2 se muestra dicha representación gráfica para los parámetros de calidad fermentativa estudiados. Los resultados obtenidos mostraron una alta correlación entre los valores predichos mediante NIRS y los valores de referencia, para todos los parámetros estudiados, con valores del coeficiente de determinación $\left(\mathrm{R}^{2}\right)$ de la regresión superiores a 0.90 y los valores de la pendiente de la regresión oscilaron entre 0.98 y 1.01, confirmando la elevada precisión de las ecuaciones desarrolladas, con valores próximos a 1 en ambos $\operatorname{casos}^{(26)}$. 
Figura 2: Valores de referencia versus predichos por NIRS para todos los parámetros fermentativos
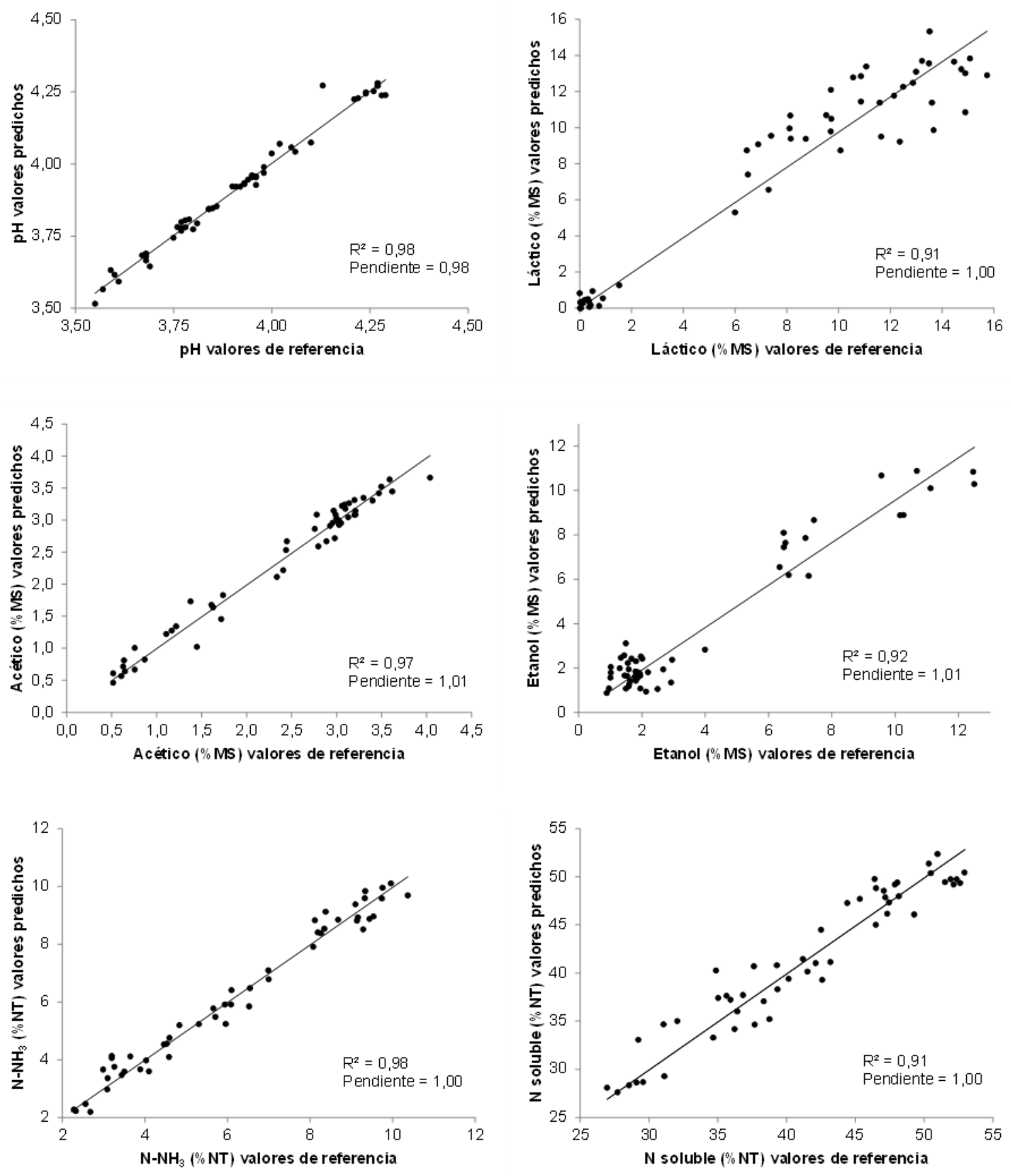

Los valores de referencia de los parámetros estudiados están distribuidos en todos los intervalos de concentración y en un rango muy amplio de variación. En el caso de la concentración de ácido láctico, los valores analíticos de referencia presentan un rango muy amplio de variación, pero no están distribuidos en todos los intervalos de concentración (Figura 2), situándose la mayoría de las muestras en el intervalo comprendido entre 6 y 15.7 \% MS, y sólo un reducido número de muestras se sitúan entre valores de 0 y 2 \% MS. 
Estos bajos contenidos en ácido láctico están en relación con la aplicación de ácido fórmico a los ensilados ${ }^{(27)}$.

Este trabajo debe considerarse de carácter preliminar al tener una cantidad limitada de muestras, siendo deseable incrementar la base de datos en futuros estudios ${ }^{(18)}$. Es recomendable la incorporación de nuevas muestras representativas, con valores que se distribuyan en los sectores menos representados, principalmente para el contenido de ácido láctico, el aumento del número de muestras del colectivo de calibración reforzará e incrementará la robustez de los modelos desarrollados ${ }^{(18)}$.

Se concluye que la tecnología NIRS, aplicada a muestras secas y molidas, es una herramienta útil y apropiada para la predicción de los parámetros de la calidad fermentativa de ensilados de girasol, siendo por tanto una alternativa para la determinación de estos parámetros con respecto a los métodos analíticos convencionales.

\section{Agradecimientos y conflictos de interés}

Trabajo financiado por los proyectos ATT 2016/106, ATT 2017/180 y 2017/182 de la Consejería del Medio Rural de la Xunta de Galicia. Se agradece al Consejo Nacional de Ciencia y Tecnología de México por otorgar becas Mixtas a Sainz-Ramírez, Plata-Reyes, Gómez-Miranda y González-Alcántara y de estancia posdoctoral a Prospero-Bernal. Los autores señalan la ausencia de conflictos de interés.

\section{Literatura citada:}

1. Demarquilly C. Composition chimique, caractéristiques fermentaires, digestibilité et quantité ingérée des ensilages de fourrages: modifications par rapport au forage vert initial. Annales de Zootechnie 1973;(22):1-35.

2. McDonald P. Silage fermentation. In : Forage Conservation in the 80's. E.G.F. Occasional Sympossium no 11. British Grassland Society. Brighton, Reino Unido. C. Thomas 1979:6775.

3. Woolford MK. The silage fermentation. Nueva York, EEUU. Marcel Dekker, Inc. 1984:350.

4. Park RS, Agnew RE, Gordon FJ, Barnes RJ. The development and transfer of undried grass silage calibrations between near infrared reflectance spectroscopy instruments. Anim Feed Sci Technol 1999;(78):325-340.

5. Givens DI, De Boever JL, Deaville ER. The principles, practices and some future applications of near infrared spectroscopy for predicting the nutritive value of foods for animals and humans. Nutr Res Rev 1997;(10):83-114. 
6. Sorensen LK. Prediction of fermentation parameters in grass and corn silage by near infrared spectroscopy. J Dairy Sci 2004;(87):3826-3835.

7. Park R, Agnew E, Porter M. Recent developments in methods to characterize the chemical and biological parameters of grass silage. In Park R, Stronge M. editors. Silage production and utilization. Proc XIV Int Silage Conf, a satellite workshop of the XX Int Grassland Cong. Belfast, Northern Ireland: Wageningen Academic Publishers; 2005:109-119.

8. Castro P. Use of near infrared reflectance spectroscopy (NIRS) for forage analysis. Lowland and grasslands of Europe: Utilization and development. Corporate Document Repository. FAO- Food And Agriculture Organization Of The United Nations, Rome, Italy. 2002. http://www.fao.org/DOCREP/006/AD236E/ad236e14.htm. Accessed July 1, 2019.

9. Pereira-Crespo S, Fernández-Lorenzo B, Resch C, Valladares-Alonso J, González L, Dagnac T, et al. Predicción de la calidad fermentativa de ensilados de hierba mediante NIRS sobre muestras secas y molidas. Pastos y Forrajes en el siglo XXI. Palma de Mallorca, España, Sociedad Española para el Estudio de los Pastos. 2015:161-167.

10. Schneiter AA, Miller JF. Description of sunflower growth stages. Crop Sci 1981;(21):901-903.

11. Castro P. Efecto de tres temperaturas de secado sobre la composición química de forrajes y heces. En: Consejería de Agricultura, Ganadería y Desarrollo Rural Ed. Actas de XXXVI Reunión Científica de la SEEP. La Rioja, España: Sociedad Española para el Estudio de los Pastos. 1996:365-368.

12. WIN ISI 1.5. ISI WINDOWS. Near-Infrared Software, The Complete Software Solution for Routine Analysis, Robust Calibration and Networking, Port Matilda, PA, USA. ISI (Infrasoft International), LLC, 2000.

13. Shenk JS, Westerhaus MO. Population definition, sample selection, and calibration procedures for near infrared reflectance spectroscopy. Crop Sci 1991;(31):469-474.

14. Barnes RJ, Dhanoa MS, Lister SJ. Standard normal variate transformation and Detrending of near infrared diffuse reflectance spectra. Appl Spectroscopy 1989;(43):772-777.

15. Shenk J, Westerhaus M. Routine operation, calibration, development and network system management manual. Silver Spring, MD, USA NIRS Systems Inc, 1995.

16. Martens H, Naes T. Multivariate calibration by data compression. In: Williams P, Norris K. editors. Near-infrared technology in the agricultural and food industries. St. Paul, Minnesota, USA. American Association of Cereal Chemists. 1987:57-88.

17. Stern M, Endres M. Laboratory manual: Research techniques in ruminant nutrition. Department of Animal Science. Minnesota, USA. University of Minnesota. 1991. 
18. Shenk JS, Westerhaus MO. Analysis of agriculture and food products by Near Infrared Reflectance Spectroscopy. USA: Monograph, NIR Systems Inc. MD, USA. Silver Spring, 1995.

19. Williams PC, Sobering DC. How do we do it: a brief summary of the methods we use in developing near infrared calibrations. In: Davies AMC, Williams P. editors. Near Infrared Spectroscopy: The future waves. Chichester, England, United Kingdom: NIR Publications; 1996:185-188.

20. Shenk JS, Westerhaus MO. Population structuring of near infrared spectra and modified partial least squares regression. Crop Sci 1991;(31):1548-1555.

21. Shenk JS, Westerhaus MO, Calibration the ISI way. In: Davies AMC, Williams P. editors. Near Infrared Spectroscopy: The future waves. Chichester, England, United Kingdom, NIR Publications; 1996:198-202.

22. Fassio A, Gimenez A, Fernandez, Vaz Martins D, Cozzolino D. Prediction of chemical composition in sunflower whole plant and silage (Helianthus annus L.) by Near Infrared Reflectance Spectroscopy. J Near Infrared Spec 2007;15(3):201-207.

23. Park H, Hoon Lee S, Cheol Lim Y, Seo S, Choi K, Hea Kim J, et al. Prediction of the chemical composition of fresh whole crop barley silages by Near Infrared Spectroscopy. J Kor Grassl Forage Sci 2013;33(3):171-176.

24. Park H, Hoon Lee S, Choi K, Cheol Lim Y, Hea Kim J, Won Lee K, et al. Prediction of the chemical composition and fermentation parameters of winter rye silages by Near Infrared Spectroscopy. J Kor Grassl Forage Sci 2014;34(3):209-213.

25. Park RS, Agnew RE, Gordon FJ, Steen RWJ. The use of near infrared reflectance spectroscopy (NIRS) on undried samples of grass silage to predict chemical composition and digestibility parameters. Anim Feed Sci Technol 1998;72(1):155-167.

26. Shenk JS, Westerhaus MO, Abrams SM 1989. Protocols for NIRS calibration: monitoring analysis results and recalibration. In Near Infared Reflectance Spectroscopy (NIRS): Analysis of forage quality. Marten GC, Shenk J, Barton FE editors. USDA. ARS. Agriculture Handbook N $N^{\circ}$ 643, Washington, USA. 1989:104-110.

27. Sainz-Ramírez A, Botana A, Pereira-Crespo S, González-González L, Veiga M, Resch $\mathrm{C}$, et al. Efecto de la fecha de corte y del uso de aditivos en la composición química y calidad fermentativa de ensilado de girasol. Rev Mex Cienc Pecu 2020;11(3):620-637. 